\title{
Symmetry Breaking in Biology
}

\author{
Rong $\mathrm{Li}^{1}$ and Bruce Bowerman² \\ ${ }^{1}$ Stowers Institute for Medical Research, 1000 East 50th Street, Kansas City, Missouri 64110 \\ ${ }^{2}$ Institute of Molecular Biology, University of Oregon, 1370 Franklin Boulevard, Eugene, Oregon 97403 \\ Correspondence: rli@stowers.org
}

Tyger! Tyger! burning bright

In the forests of the night,

What immortal hand or eye

Could frame thy fearful symmetry?

- William Blake

\section{SYMMETRY AND SYMMETRY BREAKING IN BIOLOGY}

Ymmetry in nature is often beautiful and awe Sinspiring, as William Blake famously wrote in his poem "The Tyger". One might interpret Blake's awe as stemming from the mirror-image symmetry and bilateral stripes of the tiger, and the impression of balance and power these patterns convey. In physics, however, symmetry means uniformity or invariance, or "the existence of different viewpoints from which the system appears the same" (Anderson 1972). Symmetry breaking is the process by which such uniformity is broken, or the number of points to view invariance are reduced, to generate a more structured and improbable state. Symmetry breaking, which may occur at multiple levels (see the following discussion), is a prevalent process in biology, because organismal survival depends critically on well-defined structures and patterns at both microscopic and macroscopic scales-indeed, patterns like those seen on the fearsome tiger are consequences of broken symmetry.

P.W. Anderson, Nobel Laureate in physics, speculated that increasing levels of broken symmetry in many-body systems (systems of many interacting components) correlates with increasing complexity and functional specialization (Anderson 1972). This is certainly true in biology, as symmetry breaking along welldefined axes is intimately linked to functional diversification on every scale, from molecular assemblies, to subcellular structures, to cell types themselves, tissue architecture, and embryonic body axes. For example, symmetry breaking in the macromolecular assembly of dynamic actin filaments results in asymmetric generation of the protrusive force that underlies directional cell motility (see Mullins 2009; van der Gucht and Sykes 2009; Wang 2009). Symmetry breaking at the subcellular level can lead to the establishment of a persistent polarity of growth to generate the distinct cell shapes required for such processes as cell division, cell fusion, and axon specification (see Dworkin 2009; Chang and Martin 2009; Slaughter et al. 2009; Tahirovic and Bradke 2009). In epithelial cells, the establishment of apical-basal polarity generates two surfaces of an epithelium with different components and functions, whereas asymmetry across the apical surface,

Editors: Rong Li and Bruce Bowerman

Additional Perspectives on Symmetry Breaking in Biology available at www.cshperspectives.org

Copyright (C) 2010 Cold Spring Harbor Laboratory Press; all rights reserved; doi: 10.1101/cshperspect.a003475

Cite this article as Cold Spring Harb Perspect Biol 2010;2:a003475 
called planar cell polarity, is critical for organization and pattern formation at the levels of tissues and organs (see Nelson 2009; Vladar et al. 2009).

The origin of large-scale asymmetry in biology often lies in asymmetries at a smaller scale. For example, cytoskeletal polymers, especially actin filaments and microtubules, are intrinsically polar owing to their subunit structure and assembly chemistry (see Mullins 2009). This polarity of cytoskeletal filaments plays key roles in symmetry breaking at the cellular level through a variety of mechanisms, such as protrusive force generation and directional transport of molecular assemblies and organelles. Although cytoskeletal molecules are best understood in eukaryotic organisms, recent work has led to the exciting realization that cytoskeletal molecules structurally resembling actin and tubulin also play roles in polarization and asymmetric cell division in prokaryotic cells (see Dworkin 2009). Cell polarity, referring to the property of having two distinct poles as a result of asymmetry along a particular axis, in turn, is the building block or initial state that determines asymmetry at the tissue or organismal levels. One fascinating example, left-right anatomical asymmetry in vertebrates, involves asymmetries in the molecular composition, positioning, and orientation of subcellular structures called nodal cilia, which can generate a flow of the extracellular fluid across a tissue that ultimately results in anatomical left-right body asymmetry (see Hirokawa et al. 2009). The directionality of cilia rotation and fluid flow are determined by the structure of the microtubule-motor protein complex within the cilia, and on the asymmetric positioning and tilting of cilia on the surface of nodal epithelial cells. Although symmetry breaking of larger and more complex systems can be connected to asymmetries on small scales, as the physicist predicts, "we expect to encounter fascinating and ... very fundamental questions at each stage in fitting together less complicated pieces into the more complicated system and understanding the basic new types of behavior which can result" (Anderson 1972). Similarly, we anticipate that each of the systems discussed in the articles referred to here will have both general and unique insights to offer.

\section{MODEL SYSTEMS TO STUDY BIOLOGICAL SYMMETRY BREAKING}

Opportunities to study symmetry breaking in biology abound, because it is fundamental to every physiological process. In fact, it may be argued that all qualitative cellular transitions and cellular decision-making are forms of symmetry breaking, though we focus here specifically on spatial asymmetries. Important insights into symmetry breaking have come from studies of cell division, especially in the context of asymmetric divisions that lead to different cell fates (see Munro and Bowerman 2009; Prehoda 2009; Yamashita 2009; Petricka et al. 2009), from studies of cell motility (Wang 2009), from studies of morphogenesis at the single-cell level (Dworkin 2009; Chang and Martin 2009; Slaughter et al. 2009; Tahirovic and Bradke 2009), or in multicellular structures such as polarized epithelia (Nelsom 2009; Vladar et al. 2009), and from studies of embryonic body axis formation in nematodes (Munro and Bowerman 2009), insects (Roth and Lynch 2009), and mammals (Hirokawa et al. 2009).

Work in model systems such as yeast, Caenorhabditis elegans, and Drosophila first began to reveal the molecular components that control cellular asymmetries. In addition to cytoskeletal systems with intrinsic structural polarity, certain regulatory modules also appear to have highly conserved roles in symmetry breaking. One example involves a system of molecular interactions surrounding the Cdc42 small GTPase, which orchestrates the establishment of cell polarity in many well-studied systems, including yeast cells, Drosophila neuroblasts, neurons, and epithelial cells (Chang and Martin 2009; Slaughter et al. 2009; Prehoda 2009; Wang 2009; Nelson 2009; Tahirovic and Bradke 2009). Furthermore, for reasons not yet clear, $\mathrm{Cdc} 42$ does not work alone in most of these systems but in cascades with other GTPases, such as other members of the Rho GTPase family or heterotrimeric G proteins. The versatility of $\mathrm{Cdc} 42$ and its 
GTPase cohort in diverse symmetry-breaking processes lies in their versatile regulation by a vast number of factors that control the dynamic cycling of GTPases between the GTPand GDP-bound states, and in their impressive array of effectors that regulate cytoskeleton organization, membrane trafficking, and even cell cycle progression.

Another example of a widely conserved cell polarity module is that constituted by Par-3, Par-6, and aPKC proteins, first discovered in C. elegans as critical for generating the anterior-posterior body axis (see McCaffrey and Macara 2009; Munro and Bowerman 2009). The Par3/Par6/aPKC module exists only in animal cells but not in yeast or plants as far as we know, and it works closely with Cdc42 and Rho family GTPases. The differences in cellular physiology between these organisms may lend clues to the key roles this regulatory module plays in symmetry breaking. For example, one difference lies in how cell surface tension is controlled in organisms with or without a cell wall. Animal cells maintain surface stability through a contractile actomyosin cortex, whereas the mechanical properties of cell wall glucans or cellulose lend surface stability to yeast and plant cells, respectively. Thus, the Par3/Par6/aPKC module might have coevolved with the actomyosin cortex to control cortical tension, which underlies the force that drives symmetry breaking as beautifully shown in C. elegans one-cell embryos (see Munro and Bowerman 2009). By contrast, walled cells modify surface tension through the localization of cell-wall-modifying enzymes, which, in the case of yeast cells, are carried on membrane vesicles and transported along cytoskeletal tracks (Orlando and Guo 2009; Chang and Martin 2009; Slaughter et al. 2009). Despite the above differences in cellular physiology or exactly which regulatory modules are present, a common principle has emerged that regulatory components, such as Rho GTPases and the Par3/Par6/aPKC module, and cytoskeletal systems both modify the other's behavior to form feedback loops that are critical for generating and maintaining asymmetries.

\section{COMMON PRINCIPLES IN BIOLOGICAL SYMMETRY BREAKING}

A book on cell polarity was published in the year 2000 (Drubin 2000) at a time when the study of cell polarity had just begun to move closer to center stage in cell and developmental biology, after many years in which the prominent focus had been on gene expression and signaling. This transition was perhaps a result of the recognition that a polarized cellular architecture is fundamental to cellular morphogenesis, cellfate differentiation, and formation of embryonic axes. In the roughly 10 years since, the field of cell polarity has flourished into the study of symmetry breaking at many levels of complexity. Many, if not most, of the key molecular players underlying symmetry breaking in multiple model systems have been identified. A combination of traditional genetics and biochemistry with modern live imaging methods has led to numerous breakthroughs in our mechanistic understanding of symmetrybreaking processes. Further, the influx of theoretical and computational approaches from mathematics and physics has begun to transform the field from one of descriptive observation to one of quantitative explanation and prediction. Most importantly, common themes have begun to emerge, highlighting fundamental and widely conserved mechanisms that govern biological symmetry breaking.

Of paramount importance are the physical and biochemical amplification systems that can break symmetry by turning local and transient signals or even noise into stable and system-level asymmetries. This understanding was not necessarily straightforward, because in most physiological situations, the asymmetric fate of a system appears to follow from certain "cues" that pre-exist before symmetry is broken. These cues are either localized signals that come from the environment, or "landmarks" inherited from the system's history. Well-known examples are gradients of signaling molecules that dictate the pattern of cell-fate specification, movement and growth, and sites of cell division, which impact the direction of growth for the progeny cells. Guided by such examples, the 
earlier explanations of cell polarity had largely emphasized the molecular basis of these cues and how their signals are propagated. These cues, though important in their physiological contexts, are not necessarily the engine that drives symmetry breaking: When many systems are poised to polarize, asymmetry occurs along random axes even when the cues are removed. Albeit nonphysiological, the phenomenon of random polarization has facilitated the understanding of mechanisms that amplify stochastic fluctuations or small and transient initial asymmetries provided by external cues, into more stable system-wide asymmetries. At the heart of various amplification mechanisms are positive feedback loops, resulting from intrinsic biochemical and mechanical interactions between regulatory molecules and structural (cytoskeletal and membrane) components (see Mullins 2009; van der Gucht and Sykes 2009; Slaughter et al. 2009; Munro and Bowerman 2009; Wang 2009).

This shift of focus does not ignore the much studied roles of extrinsic and intrinsic cues, and is in fact more of a return to classical ideas, articulated, for example, in D'Arcy Thompson's remarkable book "On Growth and Form," originally published in 1942. As he envisioned, "... if the symmetry be ever so little disturbed, and the shape be ever so little deformed, then there will be forces at work tending to increase the deformation, and others tending to... restore the spherical symmetry, and it can be shown that such agencies are within the range of chemistry of the cell" (Thompson 1942). Nevertheless, Thompson never overlooked the impact of the environment: "... we have begun with an initial system of very slight complexity, whose structure in no way foreshadows the result, and whose comparatively simple intrinsic forces only play their part by complex interactions with the equally simple forces of the surrounding medium." A modern take on this thought is that symmetry breaking is a result of the interplay between the system dynamics and the internal or external cues that initiate and/or orient the eventual outcome. Hence, if Thompson had lived to learn what we know today, his response might well have been a satisfied, but perhaps unimpressed, "I told you so."
But maybe Thompson would have been impressed, as he could deduce only from first principles, the laws of physics and chemistry. Our most important progress is perhaps a revitalization of these classical ideas on the basis of modern molecular exploration. Although Thomson could evoke only forces of unknown nature, we now know that, for example, it is the directional assembly of cytoskeletal polymers, the molecular motors that drive various kinds of movements, and the regulatory actions of GTPases, kinases, and proteases that constitute the key "agencies" that control cellular symmetry breaking. We are just beginning to understand symmetry breaking as the dynamic consequence of systems of molecular interactions operating in the physical context of living cells and their immediate environments.

Although it is expected that many exciting insights remain to be gained from studies of molecular pathways and biochemical mechanisms, it is clear that computational modeling has an increasingly important role in our attempts to decipher the fundamental and yet complex interactions that underlie symmetry breaking and its downstream physiological consequences. Mathematical models have already contributed greatly to our understanding of qualitative principles, e.g., the importance of stochastic fluctuations and feedback. However, turning these qualitative insights into definitive quantitative explanations of specific symmetrybreaking mechanisms will require substantial advances in our ability to quantitatively assess the concentration of molecules present in cells and their dynamic interactions with each other, using live-cell microscopy. In conclusion, asymmetry in biology is as rich and diverse as it is in the inorganic universe; As such, the study of symmetry breaking will remain a fundamental and exciting area of research for many years to come.

\section{ACKNOWLEDGMENTS}

We thank Chris Doe, Edwin Munro, and Richard Sever for critical comments and helpful suggestions on the manuscript, and Jonathan Dworkin for suggesting the opening poetry. 


\section{REFERENCES}

Anderson PW. 1972. More is different. Science 177: 393-396.

Chang F, Martin SG. 2009. Shaping fission yeast with microtubules. Cold Spring Harb Perspect Biol 1: a001347.

Drubin DG, ed. 2000. Cell polarity. Oxford University Press, Oxford.

Dworkin J. 2009. Cellular polarity in prokaryotic organisms. Cold Spring Harb Perspect Biol 1: a003368.

Hirokawa N, Tanaka Y, Okada Y. 2009. Left-Right determination: Involvement of molecular motor KIF3, cilia, and nodal flow. Cold Spring Harb Perspect Biol 1: a000802.

McCaffrey LM, Macara IG. 2009. Widely conserved signaling pathways in the establishment of cell polarity. Cold Spring Harb Perspect Biol 1: a001370.

Mullins D. 2009. Symmetry breaking in biology. Cold Spring Harb Perspect Biol 2: a003392.

Munro E, Bowerman B. 2009. Cellular symmetry breaking during C. elegans development. Cold Spring Harb Perspect Biol 1: a003400.

Nelson WJ. 2009. Remodeling epithelial cell organization: Transitions between front-rear and apical-basal polarity. Cold Spring Harb Perspect Biol 1: a000513.

Orlando K, Guo W. 2009. Membrane organization and dynamics in cell polarity. Cold Spring Harb Perspect Biol 1: a001321.
Petricka JJ, Van Norman JM, Benfey PN. 2009. Symmetry breaking in plants: molecular mechanisms regulating asymmetric cell divisions in Arabidopsis. Cold Spring Harb Perspect Biol 1: a000497.

Prehoda KE. 2009. Polarization of Drosophila neuroblasts during asymmetric division. Cold Spring Harb Perspect Biol 1: a001388.

Roth S, Lynch JA. 2009. Symmetry breaking during Drosophila oogenesis. Cold Spring Harb Perspect Biol 1: a001891.

Slaughter BD, Smith SE, Li R. 2009. Symmetry breaking in the life cycle of the budding yeast. Cold Spring Harb Perspect Biol 1: a003384.

Tahirovic S, Bradke F. 2009. Neuronal polarity. Cold Spring Harb Perspect Biol 1: a001644.

Thompson DAW. 1942. On growth and form. Cambridge University Press, Cambridge.

Van der Gucht J, Sykes C. 2009. Physical model of cellular symmetry breaking. Cold Spring Harb Perspect Biol 1: a001909.

Vladar EK, Antic D, Axelrod JD. 2009. Planar cell polarity signaling: the developing cell's compass. Cold Spring Harb Perspect Biol 1: a002964.

Wang F. 2009. The signaling mechanisms underlying cell polarity and chemotaxis. Cold Spring Harb Perspect Biol 1: a002980.

Yamashita YM, Yuan H, Cheng J, Hunt AJ. 2009. Polarity in stem cell division: asymmetric stem cell division in tissue homeostasis. Cold Spring Harb Perspect Biol 2: a001313. 


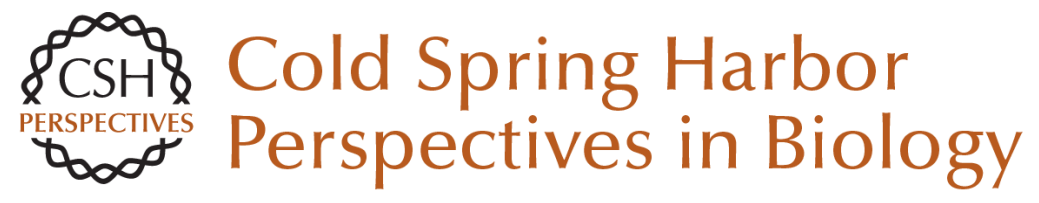

\section{Symmetry Breaking in Biology}

Rong Li and Bruce Bowerman

Cold Spring Harb Perspect Biol 2010; doi: 10.1101/cshperspect.a003475 originally published online September 23, 2009

\section{Subject Collection Symmetry Breaking in Biology}

Cytoskeletal Mechanisms for Breaking Cellular Symmetry

R. Dyche Mullins

Symmetry Breaking in Biology

Rong Li and Bruce Bowerman

Planar Cell Polarity Signaling: The Developing

Cell's Compass

Eszter K. Vladar, Dragana Antic and Jeffrey D. Axelrod

Cellular Polarity in Prokaryotic Organisms Jonathan Dworkin

Symmetry Breaking in Plants: Molecular Mechanisms Regulating Asymmetric Cell

Divisions in Arabidopsis Jalean J. Petricka, Jaimie M. Van Norman and Philip N. Benfey

The Signaling Mechanisms Underlying Cell

Polarity and Chemotaxis Fei Wang

Polarization of Drosophila Neuroblasts During Asymmetric Division Kenneth E. Prehoda

Physical Model of Cellular Symmetry Breaking Jasper van der Gucht and Cécile Sykes
Polarity in Stem Cell Division: Asymmetric Stem

Cell Division in Tissue Homeostasis

Yukiko M. Yamashita, Hebao Yuan, Jun Cheng, et al.

Symmetry Breaking in the Life Cycle of the

Budding Yeast

Brian D. Slaughter, Sarah E. Smith and Rong Li

Neuronal Polarity

Sabina Tahirovic and Frank Bradke

Membrane Organization and Dynamics in Cell

Polarity Kelly Orlando and Wei Guo

Cellular Symmetry Breaking during Caenorhabditis elegans Development Edwin Munro and Bruce Bowerman

Symmetry Breaking During Drosophila Oogenesis Siegfried Roth and Jeremy A. Lynch

Widely Conserved Signaling Pathways in the Establishment of Cell Polarity Luke Martin McCaffrey and lan G. Macara

Shaping Fission Yeast with Microtubules Fred Chang and Sophie G. Martin

For additional articles in this collection, see http://cshperspectives.cshlp.org/cgi/collection/

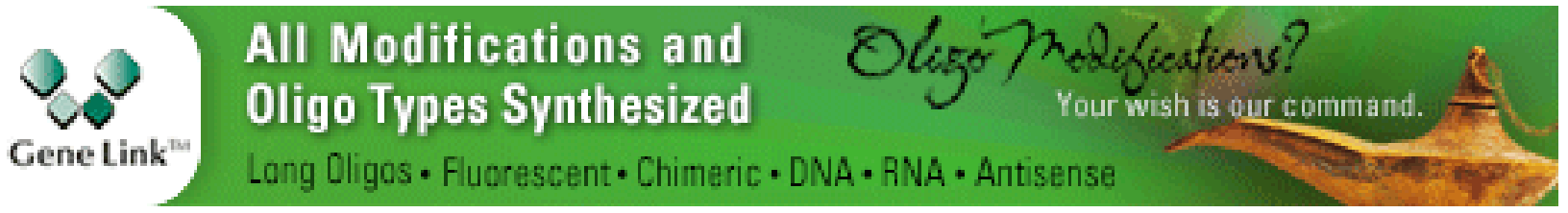

